\title{
THE SIZE OF THE SET OF LEFT INVARIANT MEANS ON AN ELA SEMIGROUP
}

\author{
ALAN L. T. PATERSON
}

\begin{abstract}
Let $S$ be an ELA semigroup and let $m(S)$ be the smallest possible cardinality of the set $\{s \in S: F s=\{s\}\}$ as $F$ ranges over the finite subsets of $S$. The main purpose of this note is to show that if $\mathrm{m}(S)$ is infinite, then $S$ has exactly $2^{2^{m(S)}}$ (multiplicative) left invariant means.
\end{abstract}

A semigroup $S$ is said to be extremely left amenable (ELA) if there exists a multiplicative left invariant mean on $S$. (See [7], [3], [4], [5].) We denote by $\varrho_{m}(S)(\mathcal{L}(S)$ ) the set of multiplicative left invariant means (left invariant means) on $S$. The cardinality of a set $X$ is denoted by $|X|$.

Investigations into the sizes of the sets of left invariant means on an infinite, left amenable, cancellative semigroup and on an amenable locally compact group are made in [1], [2], [8], [9], [10] and [6]. In particular, Chou shows in [2] that the cardinality of the set of left invariant means on an infinite, discrete, amenable group is $2^{2^{|G|}}$. Our aim is to prove a limilar result for the ELA semigroup $S$.

The family of finite subsets of a set $X$ is denoted by $\mathscr{F}(X)$. If $F \in \mathscr{F}(S)$, we set

$$
Z_{F}=\{s \in S: F s=\{s\}\} .
$$

By [3, p. 185], $Z_{F}$ is never empty. It follows that if

$$
\mathfrak{m}(S)=\min \left\{\left|Z_{F}\right|: F \in \mathscr{F}(S)\right\}
$$

then $\mathfrak{m}(S)>0$.

THEOREM. Let $S$ be an ELA semigroup. If $\mathrm{m}(S)$ is infinite, then

$$
\left|\mathcal{L}_{m}(S)\right|=|\mathcal{L}(S)|=2^{2^{m(S)}} .
$$

If $\mathrm{m}(S)$ is finite, then $\left|\mathfrak{L}_{m}(S)\right|=\mathfrak{m}(S)$, and $\mathcal{L}(S)$ is the convex hull in $l_{\infty}(S)^{*}$ of $\mathcal{E}_{m}(S)$ (and so has cardinality 1 if $\mathrm{m}(S)=1$, and cardinality $\mathrm{c}$ if $1<\mathrm{m}(S)$ $<\infty)$.

Proof. Let $F_{0} \in \mathscr{F}(S)$ be such that $\left|Z_{F_{0}}\right|=\mathfrak{m}(S)$. Let $Z=Z_{F_{0}}$.

Suppose firstly that $m(S)$ is finite. It follows from [5, Lemma 1] and from [4, Theorem A] that $Z=\mathcal{E}_{m}(S)$ and $\mathcal{L}(S)=\operatorname{co~} Z$ in $l_{\infty}(S)^{*}$.

Now suppose that $m(S)$ is infinite, and let $\alpha$ be the smallest ordinal of cardinality $m(S)$. We construct by transfinite recursion, a disjoint family $\left\{\boldsymbol{\theta}_{\boldsymbol{\varepsilon}}\right.$ :

Received by the editors September 12, 1977.

AMS (MOS) subject classifications (1970). Primary 43A07. 
$\varepsilon \in \alpha\}$ of subsets of $Z$ such that $\theta_{\varepsilon} \cap Z_{F} \neq \varnothing$ for all $\varepsilon \in \alpha$ and all $F \in$ $\mathscr{F}(S)$.

To this end, we well-order $\mathscr{F}(Z)=\left\{F_{\beta}: \beta \in \alpha\right\}$, and construct for each $\beta \in \alpha$, a family $\left\{\Delta_{\varepsilon}^{\beta}: \varepsilon \leqslant \beta\right\}$ of subsets of $Z$ such that the following conditions are satisfied:

(i) $\Delta_{\varepsilon}^{\beta} \cap \Delta_{\eta}^{\beta}=\varnothing$ if $\varepsilon \neq \eta$;

(ii) $\left|\Delta_{\varepsilon}^{\beta}\right| \leqslant|\beta|$ if $\beta$ is infinite, and $\Delta_{\varepsilon}^{\beta}$ is finite if $\beta$ is finite;

(iii) $Z_{F_{s}} \cap \Delta_{\varepsilon}^{\beta} \neq \varnothing$ whenever $\varepsilon \leqslant \delta \leqslant \beta$;

(iv) $\Delta_{\varepsilon}^{\beta} \subset \Delta_{e}^{\beta_{1}}$ whenever $\varepsilon \leqslant \beta \leqslant \beta_{1}$.

Suppose that $\gamma \in \alpha$ and that the families $\left\{\Delta_{\varepsilon}^{\beta}: \varepsilon \leqslant \beta\right\}$, satisfying conditions (i)-(iv), have been constructed for $\beta<\gamma$. From (ii), we have

$$
\left|\cup\left\{\Delta_{\varepsilon}^{\beta}: \varepsilon \leqslant \beta<\gamma\right\}\right| \leqslant \mathfrak{m}(S) .
$$

Let $\left\{x_{\varepsilon}^{\gamma}: \varepsilon \leqslant \gamma\right\}$ be a set of distinct points in $Z_{F_{\gamma}} \sim\left[\cup\left\{\Delta_{\varepsilon}^{\beta}: \varepsilon \leqslant \beta<\gamma\right\}\right]$ and define $\Delta_{\varepsilon}^{\gamma}=\left[\cup\left\{\Delta_{\varepsilon}^{\beta}: \varepsilon \leqslant \beta<\gamma\right\}\right] \cup\left\{x_{e}^{\gamma}\right\} \quad(\varepsilon<\gamma), \Delta_{\gamma}^{\gamma}=\left\{x_{\gamma}^{\gamma}\right\}$. Then the families $\left\{\left\{\Delta_{\varepsilon}^{\beta}: \varepsilon \leqslant \beta\right\}: \beta \leqslant \gamma\right\}$ satisfy the conditions (i)-(iv).

This completes the construction of the families $\left\{\Delta_{\varepsilon}^{\beta}: \varepsilon<\beta\right\}$ for all $\beta \in \alpha$. Now set $\theta_{\varepsilon}=\bigcup\left\{\Delta_{\varepsilon}^{\beta}: \beta \in \alpha\right\}$ for all $\varepsilon \in \alpha$. The family $\left\{\theta_{\varepsilon}: \varepsilon \in \alpha\right\}$ is disjoint. Let $F \in \mathscr{F}(S)$ and $\varepsilon \in \alpha$. We check that $\theta_{\varepsilon} \cap Z_{F} \neq \varnothing$. Let $x \in Z_{F_{0}} \cap Z_{F}$. Since $Z_{\{x\}} \subset Z_{F}$, it suffices to show that $\theta_{\varepsilon} \cap Z_{\{x\}} \neq \varnothing$. Suppose not. Then $\theta_{\varepsilon} \cap Z_{G}=\varnothing$ for all $G \in \mathscr{F}(Z)$ containing $x$. But the set of such $G$ is of cardinality $m(S)$, whereas, using (iii), the set of such $G$ has cardinality at most $|\varepsilon|<\mathfrak{m}(S)$. So $\theta_{\varepsilon} \cap Z_{F} \neq \varnothing$.

We now proceed as in [10, p. 158]. From [2, Lemma 2], there exists a collection $\left\{P_{\delta}: \delta \in \Delta\right\}$ of subsets of $\alpha$ where $|\Delta|=2^{\mathrm{m}(S)}$ and $\bigcap_{i=1}^{n} P_{\delta_{i}}^{e(i)} \neq \varnothing$ whenever $\delta_{1}, \ldots, \delta_{n}$ are distinct points of $\Delta$ and $\varepsilon(i)=1$ or $c$. (Note that $c$ denotes complementation.) For each $\delta \in \Delta$, set $X_{\delta}=\bigcup\left\{\theta_{\varepsilon}: \varepsilon \in P_{\delta}\right\}$. Let $\delta_{1}, \ldots, \delta_{n}, \zeta_{1}, \ldots, \zeta_{m}$ be distinct points of $\Delta$ and let $x_{1}, \ldots, x_{n}, y_{1}, \ldots, y_{m}$ $\in S^{1}$, where $S^{1}$ is the semigroup $S$ with identity adjoined. Then there exists $\gamma$ such that

$$
\theta_{\gamma} \subset\left(\bigcap_{i=1}^{n} X_{\delta_{i}}\right) \cap\left(\bigcap_{i=1}^{m} X_{\zeta_{i}}^{c}\right)
$$

Then if $s \in \theta_{\gamma} \cap Z_{\left\{x_{1}, \ldots, x_{n} y_{1}, \ldots, y_{m}\right\}}$, we have

$$
s \cup\left(\bigcap_{i=1}^{n} x_{i} X_{\delta_{i}}\right) \cap\left(\bigcap_{i=1}^{m} y_{i} X_{\zeta_{i}}^{c}\right)
$$

It follows that if $\varepsilon: \Delta \rightarrow\{1, c\}$, then there is a point of $\mathcal{L}_{m}(S)$ in the set $\cap\left\{\left(x \widehat{X}_{\delta}^{e(\delta)}\right): x \in S^{1}, \delta \in \Delta\right\}$, where $\hat{A}$ is the closure in $\beta S$ of a subset $A$ of $S$. So $\left|\mathscr{L}_{m}(S)\right| \geqslant 2^{2^{m(S)}}$. Using [5, Lemma 1] and $[4$, Theorem $A]$, we have $\mathcal{L}_{m}(S) \subset \beta Z$ and $\mathcal{L}(S) \subset l_{\infty}(Z)^{*}$. Since $|, 3 Z|=2^{2^{m(S)}}=\left|l_{\infty}(Z)^{*}\right|$, the desired result follows. 


\section{REFERENCES}

1. C. Chou, On the size of the set of left invariant means on a semigroup, Proc. Amer. Math. Soc. 23 (1969), 199-205.

2.

, The exact cardinality of the set of invariant means on a group, Proc. Amer. Math. Soc. 55 (1976), 103-106.

3. E. Granirer, Extremely amenable semigroups, Math. Scand. 17 (1965), 177-197.

4. __ Extremely amenable semigroups. II, Math. Scand. 20 (1967), 93-113.

5. Functional analytic properties of extremely amenable semigroups, Trans. Amer. Math. Soc. 137 (1969), 53-75.

6. M. Klawe, On the dimension of left invariant means and left thick subsets, Trans. Amer. Math. Soc. 231 (1977), 507-518.

7. T. Mitchell, Fixed points and multiplicative left invariant means, Trans. Amer. Math. Soc. 122 (1966), 195-207.

8. J. M. Rosenblatt, Invariant means and invariant ideals in $L_{\infty}(G)$ for a locally compact group G, J. Functional Analysis 21 (1976), 31-51.

9. Invariant means for the bounded measurable functions on a locally compact group, Math. Ann. 220 (1976), 219-228.

10. The number of extensions of an invariant mean, Compositio Math. 33 (1976), 147-159.

Department of MAThematics, University of AberdeEn, AberdeEn, SCOTLAND 